\title{
Theoretical Interpretation of Small-Scale Filaments of Light Originating from Moving Focal Spots*
}

\author{
Y. R. Shen and Michael M. T. Loy ${ }^{\dagger}$ \\ Department of Physics, University of California, Berkeley, California 94720 \\ and Inorganic Materials Research Division, Lawrence Radiation Laboratory, \\ Berkeley, California 94720 \\ (Received 4 December 1970)
}

\begin{abstract}
A simple theory is given to explain the many different features of small-scale filaments under various experimental conditions. It is shown that the observed filaments are the consequences of moving foci. Under appropriate conditions, a focal spot can move in the forward direction with a speed faster than light. As a result, the light pulse emitted from the filament at the end of the nonlinear medium has a much shorter duration than the input pulse, and shows a characteristic spectral broadening. Because of its high intensity, a moving focal spot also leaves behind it a temporary track of field-induced refractive index. Light trailing after the focal spot can then be partially trapped in the track. Trapping over an appreciable distance is possible if the velocity of the forward-moving focal spot is not much higher than the light velocity.
\end{abstract}

\section{INTRODUCTION}

The existence of small-scale filaments ${ }^{1}$ in selffocused light has aroused a great deal of interest among research workers. ${ }^{2}$ It was observed that under different experimental conditions, the results obtained on these filaments were often different. With a single-mode $Q$-switched laser pulse, the filaments were shown to be simply tracks of moving focal spots, ${ }^{3-5}$ in support of the model of Lugovoi and Prokhorov. ${ }^{6}$ On the other hand, the results obtained with a multimode laser ${ }^{7}$ or a mode-locked laser ${ }^{5}$ confirmed the existence of trapped filaments suggested by Chiao et al. ${ }^{8}$ In this paper, we propose to explain the various experimental observations of small-scale filaments by extending the model of moving focal spots. We suggest that, since a moving focal spot induces a temporary track of higher refractive index, the self-focused light can be partially trapped in the track. Under appropriate conditions, trapping can last over an appreciable distance in the medium. We can then picture a trapped filament as a leaky waveguide, along which energy is continuously fed in through self-focusing and l zaked out through diffraction. We show, from the model, that we can now explain satisfactorily the observed characteristic spectral broadening of light emitted from the filament. ${ }^{9}$ In Secs. II and III, the theoretical model is first developed on a semiquantitative basis, and then results under various experimental conditions are discussed.

\section{THEORY}

Let us first present a qualitative picture of our theory. If an intense focal spot moves in a nonlinear medium, it should leave behind it a track of field- induced refractive index $\Delta n$ which lasts at least over a period of the order of the relaxation time $\tau$ for the refractive-index change. We can therefore picture that there is a leaky optical waveguide of finite length moving along with the focal spot. Part of the self-focused light then gets trapped temporarily in this waveguide. If the focal spot is moving in the forward direction with a velocity close to the velocity of light, then the trapped light can remain trapped over a long distance until it runs out of steps with the moving waveguide. As a result of the induced $\Delta n$, the self-focused light traversing the medium acquires an increment of phase $\Delta \phi$. Since the focal spot moves with time, $\Delta \phi$ appears as a function of time $t$. A rapid phase modulation $\Delta \phi(t)$ would then lead to a broadened spectrum. ${ }^{10}$ This would happen if, towards the end of the nonlinear medium, the focal spot moved with a velocity close to the light velocity.

Details of the self-focusing dynamics can only be obtained by solving simultaneously the wave equation for the fields and the equation of motion for $\Delta n$. However, so far as the position of the focal spot is concerned (we assume $P \gtrsim P_{\mathrm{cr}}$, so that only one focal spot trajectory would appear ${ }^{6}$ ), the large-scale self-focusing theory ${ }^{11}$ has been proved to be successful, ${ }^{12}$ and for an input pulse much longer than the relaxation time $\tau$, the stationary theory of self-focusing is valid. ${ }^{13}$ Even if the input pulse is short compared with $\tau$, the modification will only affect our discussion quantitatively but not qualitatively. In the stationary theory, self-focusing of an input pulse yields a focal spot at a self-focusing distance $z_{f}$ which varies with time ${ }^{11}$ :

$$
z_{f}(t)=K /\left[P^{1 / 2}\left(t^{\prime}\right)-P_{\mathrm{cr}}^{1 / 2}\right],
$$



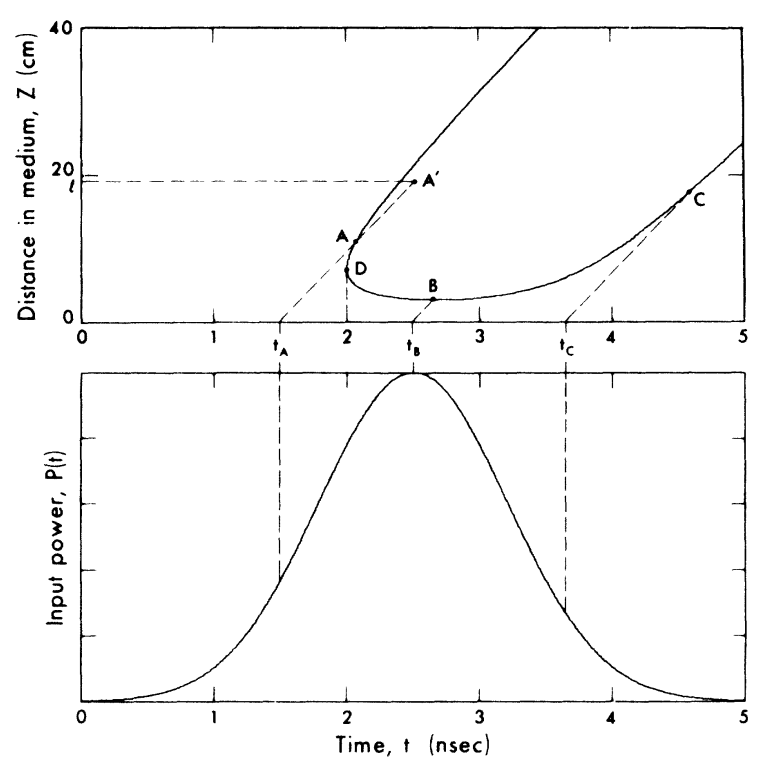

FIG. 1. Lower trace describes the input power $P(t)$ as a function of time $t$. Peak power is $42.5 \mathrm{~kW}$ and the half-width at the $e^{-1}$ point is 1 nsec. Upper trace, calculated from Eq. (1), describes the position of the focal spot as a function of time. Values of $P_{\mathrm{cr}}$ and $K$ used are $8 \mathrm{~kW}$ and $11.6 \mathrm{~cm}-(\mathrm{kW})^{1 / 2}$, respectively, which correspond roughly to an input beam of $400 \mu$ in diameter propagating in $\mathrm{CS}_{2}$. The dotted lines, with the slope equal to the light velocity in $\mathrm{CS}_{2}$, indicate how the light propagates along the $z$ axis at various times.

with $t^{\prime}=t-z_{f}(t) / c^{\prime}$, where $K$ is a parameter roughly proportional to the square of the beam diameter, $P$ is the input laser power, $P_{\mathrm{cr}}$ is the critical power for self-trapping, ${ }^{8}$ and $c^{\prime}$ is the light velocity in the medium. If $K, P_{\mathrm{cr}}$, and $P(t)$ are known, the focal-spot motion, $z_{f}$ vs $t$, can be obtained readily from Eq. (1). ${ }^{3,14}$ An example is shown in Fig. 1 for a 1-nsec input pulse (see the figure caption). The corresponding focal-spot velocity $v$ is shown in Fig. 2 as functions of $z_{f}$ and $t$, respectively. From Figs. 1 and 2, it is seen that if the variation of $P(t)$ with $t$ is sufficiently fast, then the focal spot will indeed move with a velocity close to the light velocity $c^{\prime}$. This situation is most easily realized from short input pulses.

As to the variations of $|\overrightarrow{\mathrm{E}}(r, t)|^{2}$ and $\Delta n(\overrightarrow{\mathrm{r}}, t)$ in the focal region, one must solve for the detailed self-focusing dynamics. In particular, even though the stationary theory of large-scale self-focusing predicts the position of the focal spot fairly well for a long input pulse, it would not describe correctly $|E(\overrightarrow{\mathbf{r}}, t)|^{2}$ in the small-scale focal region since here, $\Delta n$ is not likely to respond instantaneously to the variation of $|E(\overrightarrow{\mathrm{r}}, t)|^{2}$ as a result of the focal-spot motion. The transient response of $\Delta n$, together with diffraction and various types of losses, would make self-focusing of the beam more gradual in the focal region, and eventually overcome by diffraction. ${ }^{15}$ However, for our qualitative discussion later, an exact solution of $|E(\overrightarrow{\mathrm{r}}, t)|^{2}$ will not be necessary. Let us consider for the moment self-focusing of the leading part of the pulse, and consider only $|E(z, t)|^{2}$ around the focusing axis. We simply assume that as a beam self-focuses, $|E(z, t)|^{2}$ increases linearly in the prefocal region over a distance $a$, remains constant over the longitudinal dimension $2 b$ of the focal spot, and then, as a result of partial trapping or nonlinear diffraction, decreases slowly and exponentially to zero.

Then, at a fixed $z$, if we neglect the small curvature of the $U$ curve around $z$ in Fig. 1, we would have

$$
\begin{aligned}
|E(z, t)|^{2}=A\left[t-\left(t_{z}-T_{1}-T_{2}\right)\right] & \text { for } t_{z}-\left(T_{1}+T_{2}\right)<t<t_{z}-T_{2} \\
& =A T_{1} \quad \text { for } t_{z}-T_{2}<t<t_{z}+T_{2}
\end{aligned}
$$
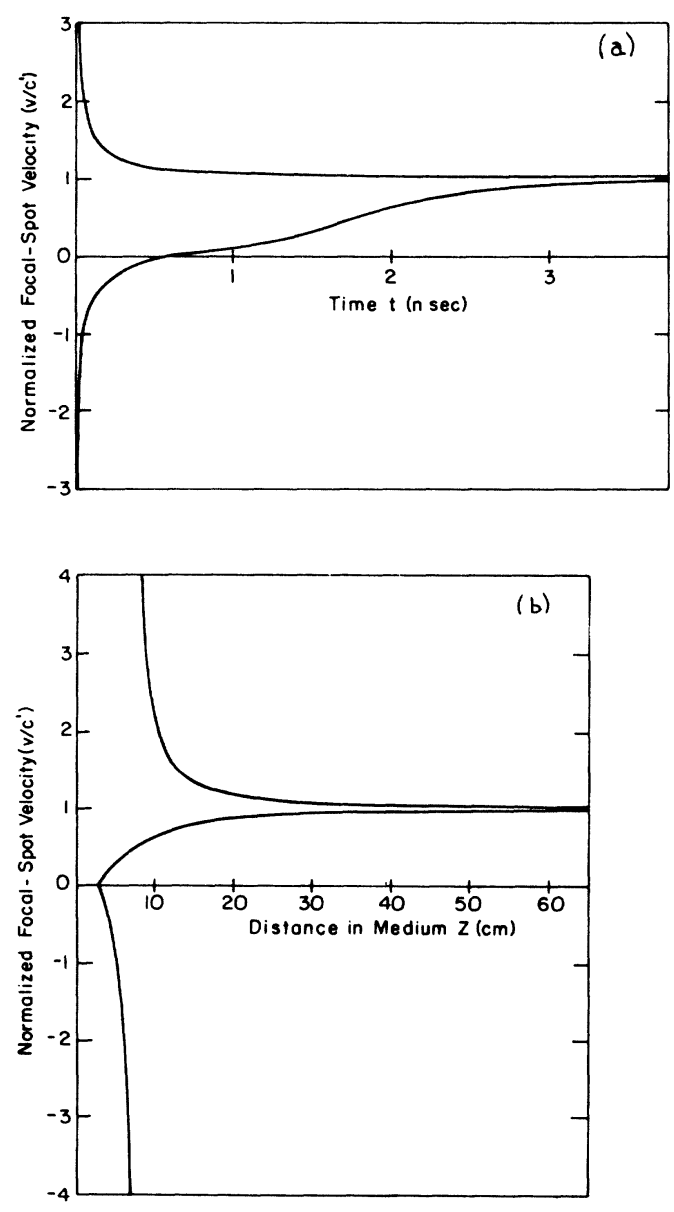

FIG. 2. Velocity of the focal spot for the case described in Fig. 1: (a) as a function of time $t$, and (b) as a function of distance $z$. 

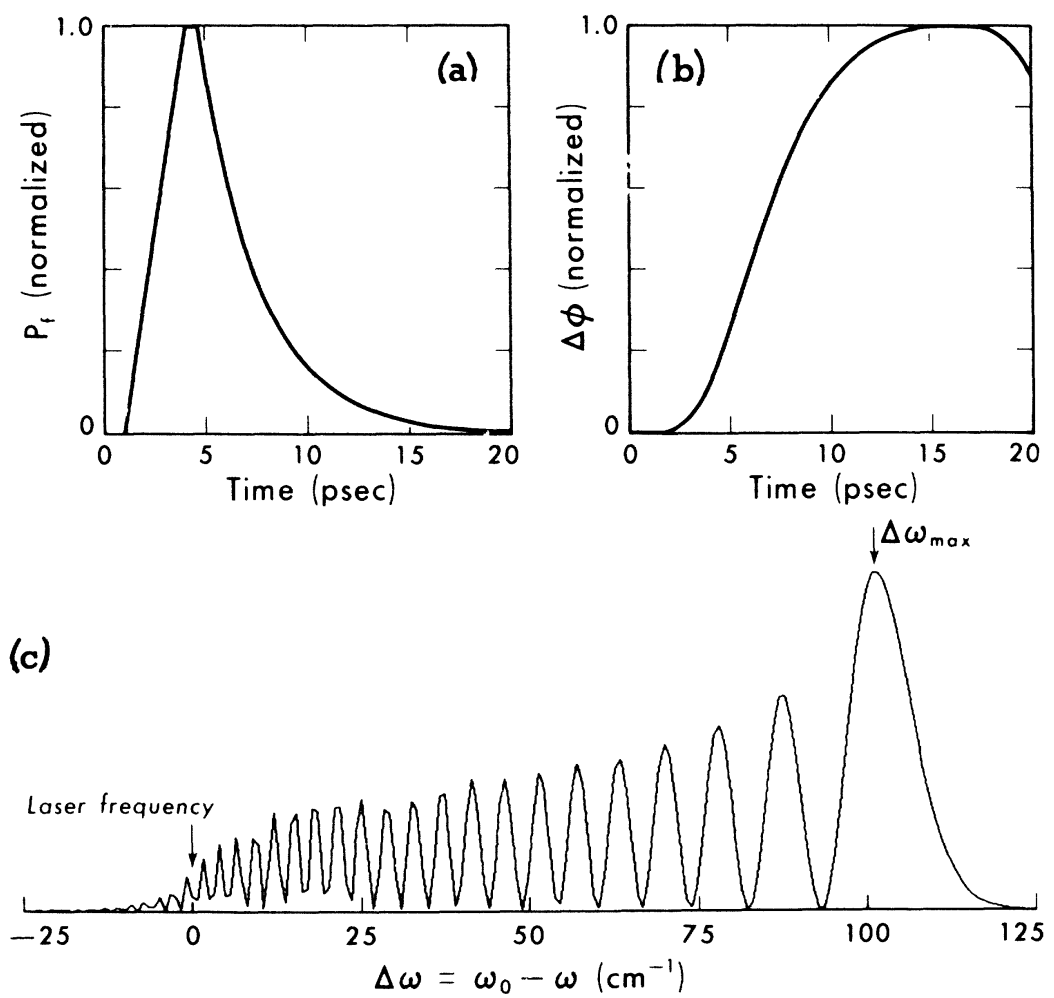

FIG. 3. (a) Normalized power $P_{f}$ of the filament pulse vs time $\left(P_{f \max }\right.$ $=11.2 \mathrm{~kW}$ ). (b) Field-induced phase change $\Delta \phi\left(\Delta \phi_{\max }=129.3 \mathrm{rad}\right)$. (c) Power spectrum of the filament pulse obtained from the Fourier transform of $P_{f}^{1 / 2} e^{i \Delta \phi}$. The curves are obtained from Eqs. (2)-(4) using the parameters $\boldsymbol{a}=0.50 \mathrm{~cm}, \boldsymbol{b}=0.05 \mathrm{~cm}$, $n_{2}=10^{-11} \mathrm{esu}, \tau=2 \times 10^{-12} \mathrm{sec}$, and $\tau^{\prime}=3 \times 10^{-12} \mathrm{sec}$ for a cell length of $l=22.5 \mathrm{~cm}$. Velocity of the focal spot at $z=l$ is $1.124 c^{\prime}$.

$$
=A T_{1} e^{-\left(t-t_{z}-T_{2}\right) / \tau^{\prime}} \quad \text { for } t>t_{z}+T_{2},
$$

with

$$
\begin{aligned}
& A=16 P / c d^{2} T_{1}, \quad T_{1}=a\left(v-c^{\prime}\right) / v c^{\prime}, \\
& T_{2}=b\left(v-c^{\prime}\right) / v c^{\prime},
\end{aligned}
$$

where $t_{z}$ is the time when the focal spot appears at $z, P$ is the input laser power at $\left(t_{z}-z / c^{\prime}\right), d$ is the diameter of the focal spot, and $v$ is the velocity of the focal spot at $z$. As one would expect, the parameters $a, b$, and $d$ should generally depend on $P(t)$, the diameter, and the spatial distribution of the input beam, and on the properties of the medium.

We should emphasize that Eq. (2) is only meant to be a crude approximation of the true pulse. However, our qualitative discussion below will not be affected by the detailed pulse shape as we shall see. There is some arbitrariness in choosing the values for $T_{1}$ and $T_{2}$ (or $a$ and $b$ ), ${ }^{16}$ but in ordinary liquids $\left(T_{1}+T_{2}\right)$ must be of the order of the relaxation time $\tau$ ( $\sim$ a few psec for orientational relaxation) for $\Delta n$. It cannot be much smaller since, from experimental observations, ${ }^{1,17}$ we know that the maximum $\Delta n(t)$ at $z$ on the axis is not much less than $\Delta n_{0}(z)=\frac{1}{2} n_{2}|E(z, t)|_{\max }^{2}$. The value of $\left(T_{1}+T_{2}\right)$ cannot be much larger either since, if $\left(T_{1}+T_{2}\right) \gg \tau$, we would expect the stationary selffocusing theory to be valid even in the focal region. but we know that when the stationary self-focusing theory holds the focal spots would be sharp with $\left(T_{1}+T_{2}\right)<\tau,{ }^{11,13}$ contrary to the original assumption. Also, we noticed in our experiments $s^{3,5}$ that the focal spots are usually well defined, suggesting that $\left(T_{1}+T_{2}\right)$ cannot be much larger than $\tau$. There is also arbitrariness in choosing a value for $\tau^{\prime}$ in Eq. (2). A larger $\tau^{\prime}$ corresponds to trapping of light over a longer duration. Self-trapping of light (i.e., trapping of light by its own instantaneous power in a nonlinear medium $)^{8}$ occurs as $\tau^{\prime}$ becomes infinite.

We now assume that $\Delta n$ obeys the simple relaxation equation

$$
\frac{\partial(\Delta n)}{\partial t}+\frac{\Delta n}{\tau}=\frac{n_{2}}{\tau}|E(t)|^{2}
$$

where $n_{2}$ is the nonlinear refractive index. With $|E(z, t)|^{2}$ given by Eq. (2), we can then solve for $\Delta n(z, t)$ around the focusing axis. Let us again refer back to Fig. 1. Consider the laser light entering the medium at $t_{A}$ and propagating in the medium along $A A^{\prime}$ in Fig. 1. Because of the induced $\Delta n$, the part of the self-focused light which trails the moving focal spot along the axis acquires a phase increment $\Delta \phi$ at $z=l$ given by

$$
\Delta \phi\left(t=t_{A}+l / c^{\prime}\right)=\int_{0}^{l}(\omega / c) \Delta n\left(z, t^{\prime}=t_{A}+z / c^{\prime}\right) d z .
$$



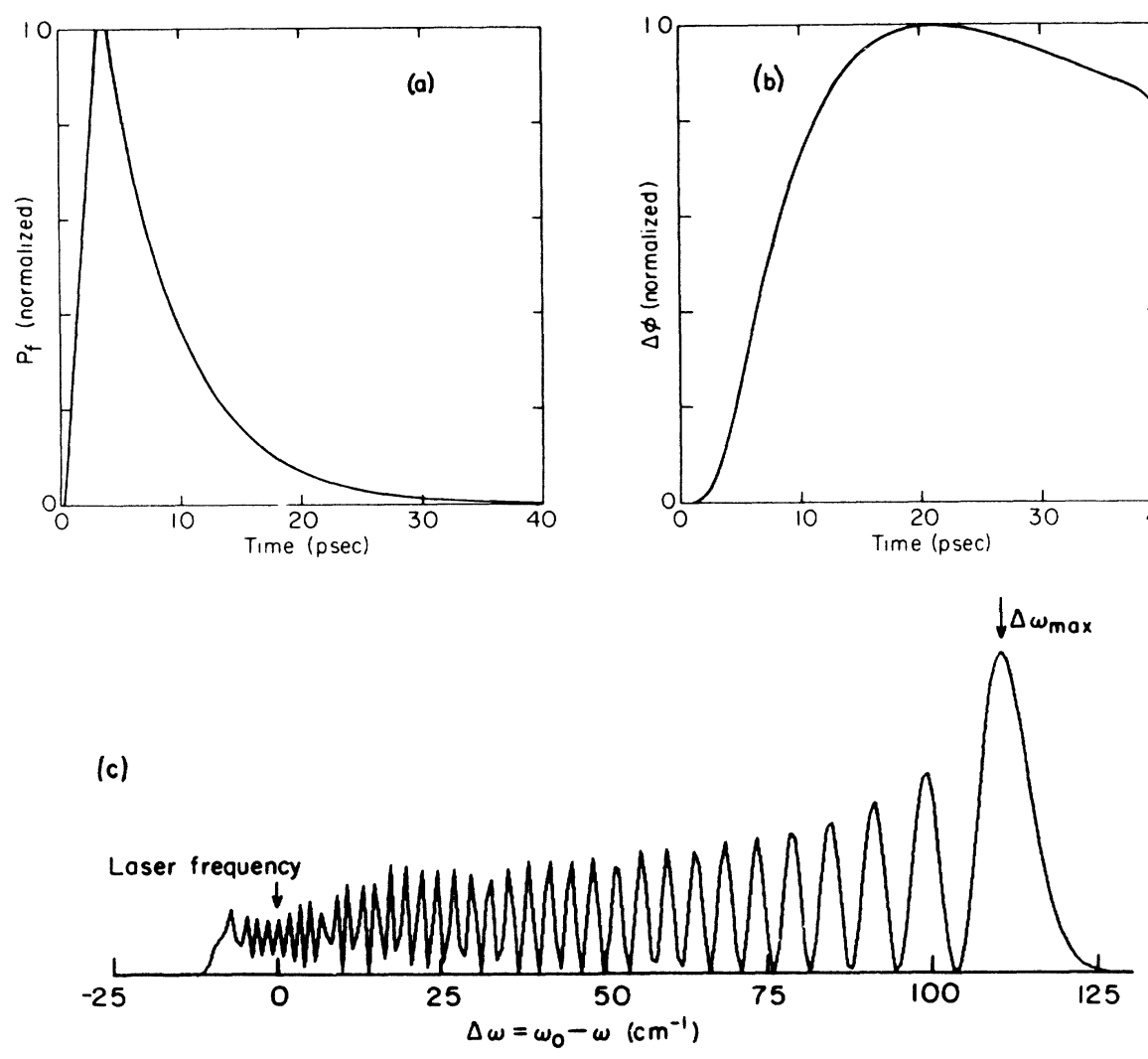

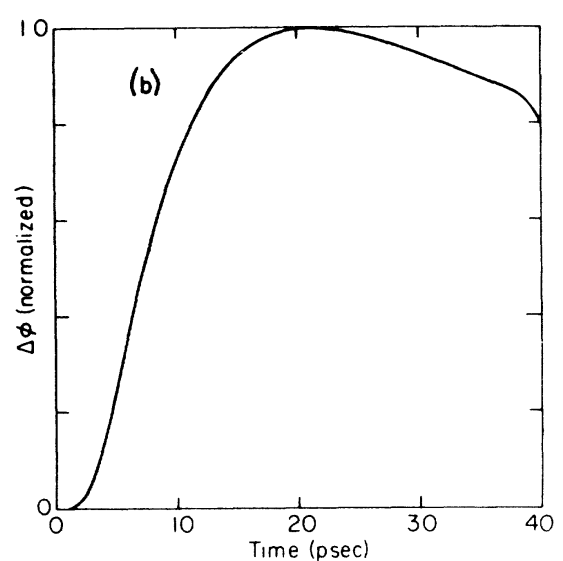

FIG. 4. Curves describing $P_{f}$ vs $t, \Delta \phi$ vs $t$, and the power spectrum of the filament pulse obtained with all parameters in Fig. 3 kept unchanged except $\tau^{\prime}=6 \times 10^{-12} \mathrm{sec}$.
From the Fourier transform of $P_{f}^{1 / 2} e^{-i \omega t+i \Delta \phi}$, where $P_{f}=\left(d^{2} c / 16\right)|E(l, t)|^{2}$, we can obtain the power spectrum of the light pulse emitted from the smallscale filament at the end of the medium.

As an example, we consider the case of propagating the 1-nsec laser pulse of Fig. 1 into a $\mathrm{CS}_{2}$ cell $22.5 \mathrm{~cm}$ long. We assume $T_{1}=1.5 \tau$ $(a=0.50 \mathrm{~cm}), T_{2}=0.15 \tau(b=0.05 \mathrm{~cm}), d=5 \mu$, $n_{2}=10^{-11} \mathrm{esu}, \tau=2 \times 10^{-12} \mathrm{sec}$, and $\tau^{\prime}=3 \times 10^{-12} \mathrm{sec}$. In Figs. 3(a) and 3(b), we have plotted $P_{f}(t)$ and $\Delta \phi(t)$ as functions of $t$, and in Fig. 3(c), the corresponding power spectrum. It is seen that the output filament pulse is about $4 \mathrm{psec}$ long, and its power spectrum has the characteristic spectral broadening and semiperiodic structure observed in experiments. ${ }^{7,9}$ The asymmetry of the $\phi(t)$ curve is reflected by the Stokes-anti-Stokes asymmetry in the spectrum. ${ }^{9,10}$ Such a spectral broadening was earlier explained by assuming the existence of a self - trapped filament with a certain definite length. ${ }^{9,10}$ It is interesting to note that in order to explain the observed spectral broadening in $\mathrm{CS}_{2}$ using the self-trapping model, Gustafson et al. ${ }^{10}$ had to assume a filament pulse with a pulse width of a few psec, which is about equal to the filament pulse width we obtained from our calculation. To see the effect of partial trapping on spectral broadening, we show in Figs. 4 and 5 the broadened spectra corresponding to $\tau^{\prime}=3 \tau$ and $\tau^{\prime}=5 \tau$, respectively, with all the other parameters kept unchanged. We also show in Fig. 6 the spectrum for the special case where we assume no trapping at all. \{The corresponding filament pulse has the exponential decay replaced by $\left.A\left[T_{1}-\left(t-t_{\varepsilon}\right)-T_{2}\right].\right\}$ It is seen that trapping affects only the detailed structure of the broadened spectrum, but it does not change appreciably the extent of spectral broadening on the Stokes side. In particular, with no trapping (Fig. 6), the semiperiodic structure in the broadened spectrum is largely smeared out. On the anti-Stokes side, however, there is more broadening if the trapping duration is longer. We have also tried other values of $T_{1}$ and $T_{2}$. Qualitatively, the phase modulation is stronger for larger values of $T_{1}$ and $T_{2}$ and, hence, the spectrum shows more broadening.

In fact, the extent of spectral broadening on the Stokes side is given approximately by $\Delta \omega_{\max }$ $\approx-(\partial \Delta \phi / \partial t)_{\max }$, when the inverse of the duration of the filament pulse is small compared with $\Delta \omega_{\max }$. To find $(\partial \Delta \phi / \partial t)_{\max }$ at $z=1$, we can approximate the upper branch of the $U$ curve in Fig. 1 by a straight line in the range of interest. Then, the light propagating along $A A^{\prime}$ in Fig. 1 reaches $z=l$ at the time $t=t_{l}+\left(l-z_{A}\right)\left(1 / c^{\prime}-1 / v\right)$, where $t_{l}$ is the time when the center of the focal spot ap- 

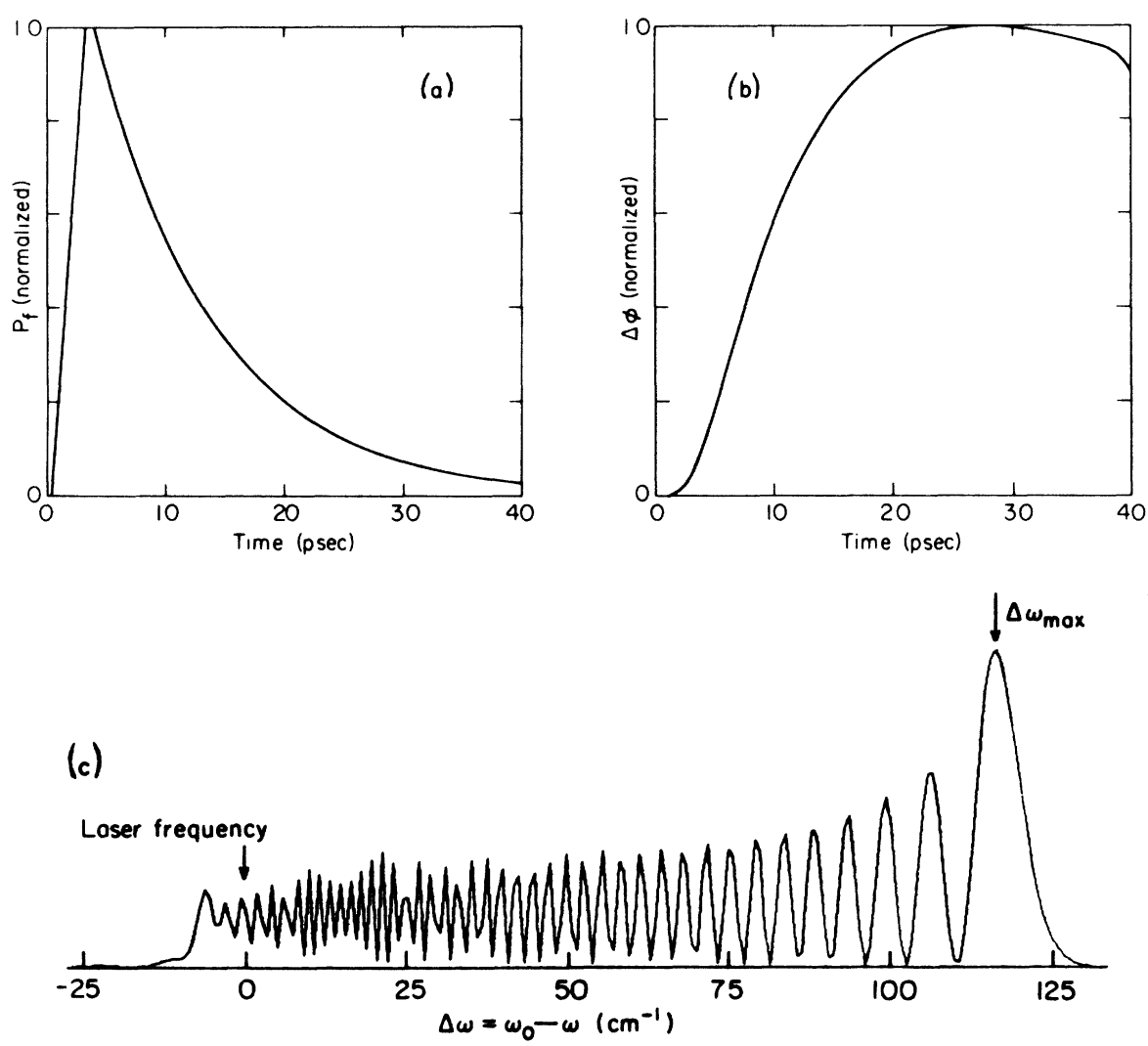

FIG. 5. Curves describing $P_{f}$ vs $t, \Delta \phi$ vs $t$, and the power spectrum of the filament pulse obtained with all parameters in Fig. 3 kept unchanged except $\tau^{\prime}=10^{-11} \mathrm{sec}$ pears at $z=l$. From Eq. (4) we now have

$$
\left(\frac{\partial \Delta \phi}{\partial t}\right)_{z=l}=\left(\frac{1}{c^{\prime}}-\frac{1}{v}\right)^{-1}\left(\frac{\partial}{\partial z_{A}}\right) \int_{z_{A^{-}(a+b)}^{l}}^{l} \frac{\omega}{c} \Delta n\left(z, z_{A}\right) d z,
$$

where the integration is along the path $A A^{\prime}$. Since the input laser power is essentially constant for the small duration of the filament pulse, $\Delta n$ is essentially independent of $z_{A}$. We then have

$$
\begin{aligned}
\Delta \omega_{\max } & \approx-\left[\left(\frac{\partial \Delta \phi}{\partial t}\right)_{z=l}\right]_{\max } \\
& =(1 / c-1 / v)^{-1}(\omega / c)\left(\Delta n_{\max }\right)_{z=l} .
\end{aligned}
$$

In Eq. (6), $\left(\Delta n_{\max }\right)_{z=l}$ is the only quantity which depends on the detailed self-focusing dynamics, but as we mentioned earlier, $\left(\Delta n_{\max }\right)_{z=l}$ should not be much less than $\Delta n_{0}=\left(\frac{1}{2}\right) n_{2}|E(l, t)|_{\max }^{2}$, which can be estimated from measurements. ${ }^{1,17}$ Spectral broadening should become more appreciable when, towards the end of the medium, the velocity $v$ of the local spot approaches the light velocity $c^{\prime}$. For $\Delta n_{\max } \sim 10^{-3}, v=1.01 c^{\prime}$, we would have $\left(\Delta \omega_{\max } / \omega\right)$ $\sim 0.1$. The above approximation breaks down if the curvature of the $U$ curve becomes important in calculating $\partial \Delta \phi / \partial t$. This happens only when the input laser pulse has a pulse width comparable with $\tau$. Note that in our case, as a result of focal-spot movement, spectral broadening arises because the length of the optical path in which $\Delta n \neq 0$ varies with time. In the earlier calculations, ${ }^{9,10}$ the optical path with $\Delta n \neq 0$ was assumed to have a constant length, and it is through the explicit dependence of $\Delta n$ on time that spectral broadening comes in.

\section{DISCUSSION}

From the model discussed here, we expect to find small-scale filaments of different characteristics under different experimental conditions. This explains the many different results reported in the literature on the subject. Figure 1 suggests that, in general, we should expect to see three different types of small-scale filaments depending on the input conditions:

(a) If the length of the medium $l$ (relative to the input laser pulse) is small such that $z_{B}<l<z_{D}$ in Fig. 1, then the filament is composed of a moving focal spot which moves from the end of the medium inward to $z_{B}$ and then moves out again, assuming that self-focusing is not affected by stimulated scattering. Little trapping would exist and essentially no spectral broadening of light emitted from the small-scale filament should be observed. Near $z_{B}$, the velocity of the moving focal spot is small. 

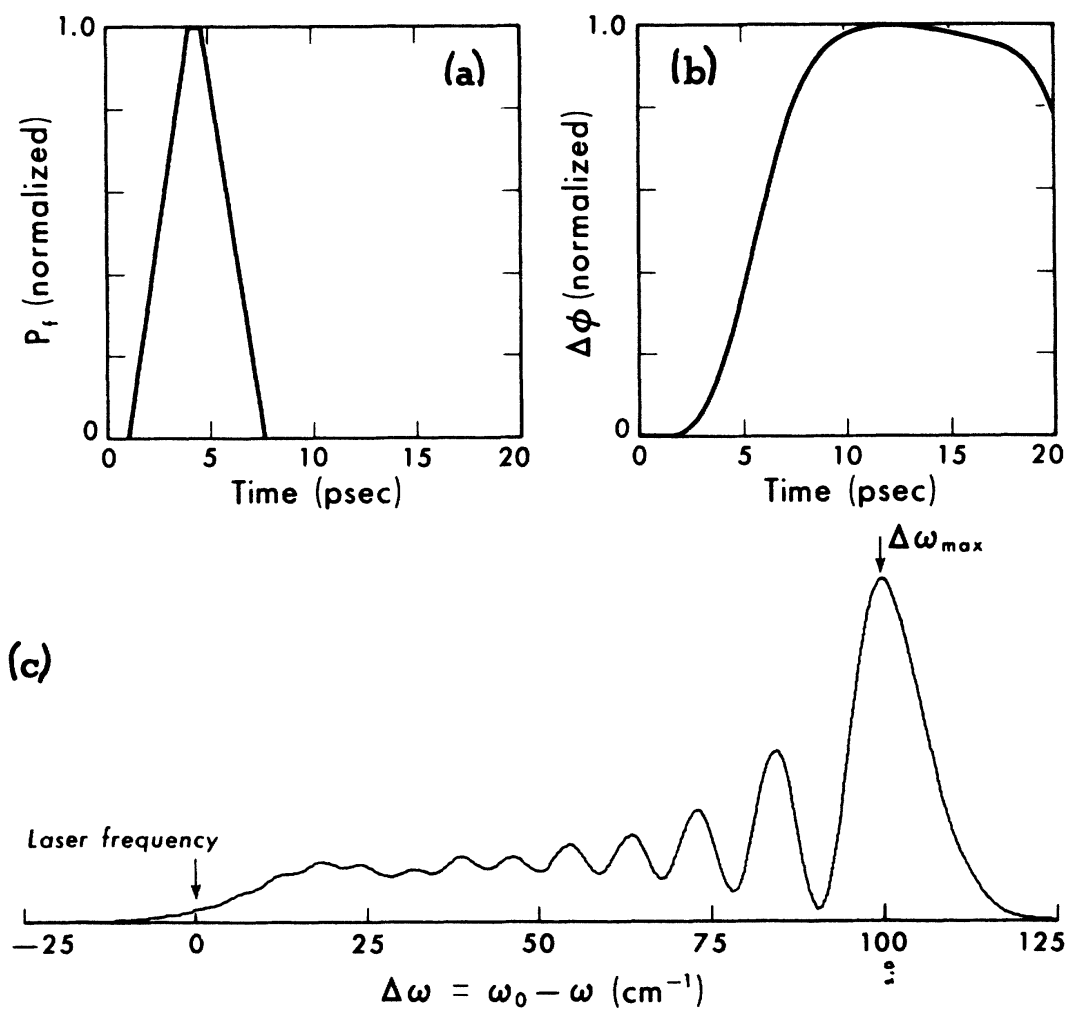

FIG. 6. Curves describing $P_{f}$ vs $t$, $\Delta \phi$ vs $t$, and the power spectrum of the filament pulse obtained with all parameters in Fig. 3 kept unchanged except that the decay of $P_{f}(t)$ is linear and symmetric to the linear rise of $P_{f}$.
Consequently, if $l \approx z_{B}$, the filament pulse emitted at the exit end becomes correspondingly longer. These predictions agree well with the experimental results obtained by a single-mode $Q$-switched laser at a power level slightly above the self-focusing threshold in the medium. ${ }^{3}$

(b) If $l>z_{D}$, but is not excessively large, the focal spot first appears at $z_{D}$ and subsequently splits into two, one moving forward and the other first moving backward to $z_{B}$ and then moving out again (see Fig. 1). In this case, since the forwardmoving focal spot has a velocity much faster than $c^{\prime}$, light cannot be trapped over any appreciable distance. The spectrum of the filament pulse emitted at the exit end would have no appreciable broadening. The spectral width does, however, increase somewhat with the length of the medium. Again, these predictions were confirmed by the experimental results obtained from a single-mode $Q$-switched laser. ${ }^{5}$

(c) If $l \gg z_{c}$, so that the velocity of the moving focal spot approaches $c^{\prime}$ towards the end of the medium, then part of the self-focused light trailing after the focal spot can be trapped over a long distance. The filament pulse emitted from the medium would probably have a duration of the order of $\tau$. Its spectrum should now have appreciable broadening which increases as the length $l$ increases. ${ }^{7}$ In usual experiments, with a reasonable length $l$, this situation is most easily realized by a short input laser pulse. We notice that filaments with large spectral broadening were observed only when a mode-locked laser ${ }^{5,18-20}$ or a multimode $Q$-switched laser ${ }^{7,9}$ was used.

So far. we have considered only self-focusing of the leading part of the input pulse. For the trailing part, it is clear from Fig. 1 that the light diffracted from the focal region will not be trapped since the moving focal spot which initiates the optical waveguide is lagging behind the light. It is also possible that the trailing part never self-focuses since the backward stimulated Raman and Brillouin scattering effectively terminate self-focusing by depleting the incoming laser power. This is the case wnen the input pulse is sufficiently long. ${ }^{2,21}$ When the input pulse is short ( $\left.\_1 \mathrm{nsec}\right)$, stimulated Brillouin scattering is largely suppressed, but stimulated Raman scattering is not. For the upper branch of the $U$ curve in Fig. 1, stimulated scattering should have little effect on self-focusing. The backward stimulated radiation originated from the focal region cannot interact with the incoming light before focusing and, therefore, cannot prevent the formation of the filament. Forward Raman radiation of high intensity is also generated in the focal region. Whenever the laser light is partially trapped over a long distance, part of the Raman radiation also gets trapped, and the corresponding Raman spec- 
trum of the filament pulse should have a broadening with the characteristic semiperiodic structure.

Although our discussion above has been limited to input pulses with duration much longer than the relaxation time $\tau$, the qualitative aspects of the theory should hold even for the case of ultrashort pulses. We realize that if the input pulse width is comparable with $\tau$, then the transient response of the medium should affect even the self-focusing distance. For the upper branch of the $U$ curve in Fig. 1, the beam with an input power $P$ should now self-focus at a distance larger than the value given by Eq. (1), but the curve should still retain its qualitative feature. Also, because of transience, the dimension of the focal spot would be larger. The transient effect on the lower branch of the $U$ curve may be even more drastic, since self-focusing of the lagging part of the input pulse may now be affected by self-focusing of the leading part. ${ }^{22}$ From our theory, we can make the following qualitative predictions for the case of ultrashort pulses. The duration of the output filament pulse should still be of the order of $\tau$. Spectral broadening with semiperiodic structure should be greatly enhanced, with Stokes and anti-Stokes broadening equally prorounced. In this case, light may also be trapped over a much longer distance. These predictions can explain qualitatively the recent experimental results obtained with picosecond mode-locked pulses. ${ }^{18,19}$ Spectral broadening due to self-focusing of picosecond pulses in rare-gas liquids and solids has also been observed. ${ }^{20}$ Distortion of local electronic clouds is supposed to be responsible for the optical nonlinearity ${ }^{20}$ and the corresponding relaxation time $\tau$ is of the order of $10^{-15} \mathrm{sec}$. Here again, although the detailed self-focusing dynamics is unknown, we can see from our model that the output filament pulse should have a duration much shorter than a picosecond. Then, the duration of the filament pulse alone can probably explain the observed spectral broadening.

It is seen that our theoretical analysis can indeed explain qualitatively the many different features of small-scale filaments observed under various experimental conditions. For more quantitative discussion, we must extend the calculation of dynamic self-focusing ${ }^{13}$ to and beyond the small-scale focal region. The results should also yield the diameter of the focal spot and hence the dimension of the small-scale filament.

\section{ACKNOWLEDGMENTS}

We would like to thank Professor C. H. Townes and Professor T. K. Gustafson for a helpful discussion.

\footnotetext{
*Work performed under the auspices of the U. S. Atomic Energy Commission.

IIB M Fellow.

${ }^{1}$ R. Y. Chiao, M. A. Johnson, S. Krinsky, H. A. Smith, C. H. Townes, and E. Garmire, IEEE J. Quantum Electron. QE-2, 467 (1966); R. C. Brewer, J. R. Lifsitz, E. Garmire, R. Y. Chiao, and C. H. Townes, Phys. Rev. 166, 326 (1968).

${ }^{2}$ See, for example, Digest of Technical Papers for the Sixth International Quantum Electronics Conference, Kyoto, Japan, 1970, pp. 150-173 (unpublished).

${ }^{3}$ M. M. T. Loy and Y. R. Shen, Phys. Rev. Letters $\underline{22}, 994$ (1969).

${ }^{4}$ G. M. Zverev, E. K. Maldutis, and V. A. Pashkov, Zh. Eksperim. i Teor. Fiz. Pis'ma v Redaktsiyu 9, 108 (1969) [Sov. Phys. JETP Letters 9 , 61 (1969)]; V. V. Korobkin, A. M. Prokhorov, R. V. Serov, and M. Ya. Shchelev, ibid. 11, 153 (1970) [ibid. 11, 94 (1970)].

${ }^{5}$ M. M. T. Loy and Y. R. Shen, Phys. Rev. Letters 25, 1333 (1970).

${ }^{6}$ A. L. Dyshko, V. N. Lugovoi, and A. M. Prokhorov, Zh. Eksperim. i Teor. Fiz. Pis'ma v Redaktsiyu $\underline{6}, 655$ (1967) [Sov. Phys. JETP Letters $\underline{6}, 146$ (1967)]; V. N. Lugovoi and A. M. Prokhorov, ibid. ㄱ, 153 (1968) [ibid. 7,117 (1968)].

${ }^{7}$ M. M. Denariez-Roberge and J-P. E. Taran, Appl. Phys. Letters 14, 205 (1969).

${ }^{8}$ R. Y. Chiao, E. Garmire, and C. H. Townes, Phys. Rev. Letters $\underline{13}, 479$ (1964).

${ }^{9}$ R. G. Brewer, Phys. Rev. Letters 19,8 (1967); F. Shimizu, ibid. 19, 1097 (1967); A. C. Cheung, D. M. Rank, R. Y. Chiao, and C. H. Townes, ibid. $\underline{20}, 786$
}

(1968).

${ }^{10} \mathrm{~F}$. Shimizu, Phys. Rev. Letters $\underline{19}, 1097$ (1967); T. K. Gustafson, J-P. E. Taran, H. A. Haus, J. R. Lifsitz, and P. L. Kelley, Phys. Rev. 177, 306 (1969).

${ }^{11}$ P. L. Kelley, Phys. Rev. Letters 15,1005 (1965).

${ }^{12}$ C. C. Wang, Phys. Rev. Letters 16 , 344 (1966).

${ }^{13}$ J. A. Fleck and P. K. Kelley, Appl. Phys. Letters 15,313 (1969).

${ }^{14}$ T. K. Gustafson and J-P. E. Taran, IEEE J. Quantum Electron. QE-5, 381 (1970).

${ }^{15}$ If an appreciable fraction of the laser power is depleted by stimulated scattering in the focal region, then the diffracted light is not likely to self-focus again as suggested by the calculation of J. H. Marburger and E. Dawes, Phys. Rev. Letters 21, 566 (1968); E. L. Dawes and J. H. Marburger, Phys. Rev. 179, 862 (1969).

${ }^{16}$ Correct values for $T_{1}$ and $T_{2}$ can be obtained only by solving the detailed self-focusing dynamics.

${ }^{17}$ F. Shimizu and B. P. Stoicheff, IEEE J. Quantum Electron. QE-5, 544 (1969).

${ }^{18}$ R. G. Brewer and C. H. Lee, Phys. Rev. Letters 21, 267 (1968); R. Polloni, C. A. Sacchi, and O. Svelto, ibid. 23, 690 (1969).

${ }^{19}$ R. L. Carmen, J. Reinjes, and F. Shimizu (private communication).

${ }^{20}$ R. R. Alfano and S. L. Shapiro, Phys. Rev. Letters 24, 584 (1970); 24 , 1217 (1970).

${ }^{21}$ M. Maier, G. Wendl, and W. Kaiser, Phys. Rev. Letters 24, 352 (1970).

${ }^{22}$ S. A. Akhmonov (private communication). 